REVUE DE L'INSTITUT

FRANÇAIS D'HISTOIRE

EN ALLEMAGNE

\section{Revue de l'IFHA}

Revue de l'Institut français d'histoire en Allemagne

$5 \mid 2013$

IFHA 5

\title{
Un musée pour toute la ville : la nouvelle conception du Musée historique de Francfort (2008-2016)
}

Jan Gerchow

Traducteur : Aude-Marie Certin

(2) OpenEdition

Journals

Édition électronique

URL : http://journals.openedition.org/ifha/7390

DOI : 10.4000/ifha.7390

ISSN : 2198-8943

Éditeur

IFRA - Institut franco-allemand (sciences historiques et sociales)

Édition imprimée

Date de publication : 1 décembre 2013

ISSN : 2190-0078

Référence électronique

Jan Gerchow, « Un musée pour toute la ville : la nouvelle conception du Musée historique de Francfort (2008-2016) », Revue de l'IFHA [En ligne], 5 | 2013, mis en ligne le 17 février 2014, consulté le 01 mai 2019. URL : http://journals.openedition.org/ifha/7390 ; DOI : 10.4000/ifha.7390

Ce document a été généré automatiquement le 1 mai 2019.

(C)IFHA 


\title{
Un musée pour toute la ville : la nouvelle conception du Musée historique de Francfort (2008-2016)
}

\author{
Jan Gerchow \\ Traduction : Aude-Marie Certin
}

\section{NOTE DE L'ÉDITEUR}

Ce texte reprend différents exposés de l'auteur tenus à Cologne (Museumsverband Rheinland, 6 juin 2011), à Francfort-sur-le-Main (KIRM, 10 novembre 2011), à Paris (Maison de l'Histoire de France, 8 juin 2012), Cracovie (Musée historique de la ville de Cracovie, 13 septembre 2012), à Vancouver (CAMOC, 26 octobre 2012), à Nuremberg (Musée de la ville de Nuremberg, 12 novembre 2012), à Zurich (Hochschule der Künste und Landesmuseum février 2013) et à Vienne (Wien Museum, 25 juin 2013).

Aude-Marie Certin. IFHA, Francfort-sur-le-Main.

1 Le musée historique d'une ville est aujourd'hui souvent compris comme un musée spécialisé, tourné vers une discipline scientifique donnée, en l'occurrence, la science historique. Ceci vaut pour tous les musées municipaux se trouvant dans une position comparable, comme les musées d'histoire de l'art, d'archéologie ou des sciences de la nature, qui, de la même façon, se rapportent à une discipline scientifique, ainsi qu'à une région ou un lieu donné. Cependant les musées historiques sont en même temps des lieux d'information et de savoir sur toute la ville : même s'ils sont parfois appelés autrement, ils sont à proprement parler des musées de la ville. Il y est question des traditions "propres » à la ville, de ses héritages culturels, souvent aussi du présent de la ville, comme de ses perspectives d'avenir. Depuis une décennie, ces musées sont l'objet d'un vif regain d'intérêt, non seulement de la part des spécialistes des musées, mais aussi du grand public. Cela est lié, d'une part, à une nouvelle façon d'envisager l'urbanisme dans 
son ensemble, y compris dans ses dimensions culturelles; d'autre part, au fait que de nombreux musées de ville des années 1970-1990 ont été l'objet d'importants travaux d'assainissement qui, bien souvent, ont rendu possible une nouvelle façon de les concevoir ${ }^{1}$.

$2 \mathrm{Au} \mathrm{XIX}^{\mathrm{e}}$ siècle ou au début du XXe siècle, les musées de ville ont été dans bien des cas les premiers musées publics. Avec la spécialisation des musées au XXe siècle, c'est souvent à partir d'eux et à partir de leurs collections, qu'ont été créés et aménagés un certain nombre d'autres musées municipaux ${ }^{2}$. Le Musée historique de Francfort est ainsi un musée de la ville de la « première heure ». Depuis sa fondation, il a toujours été un miroir de la ville de Francfort ; il s'est ainsi transformé plusieurs fois, reflétant les changements de situations de la ville elle-même. En 1861, l'avocat francfortois Friedrich Scharff proposa de créer un espace consacré à «l'histoire, aux coutumes et à l'art de Francfort ». Il le fit dans le contexte des débats autour de l'aménagement de la place de parade autour de la Hauptwache, et de l'emplacement d'un nouveau monument pour Schiller. Friedrich Scharff avait en tête l'idée d'un musée d' " histoire de la Bildung » universelle. Le projet de musée fut cependant reporté du fait de l'annexion de la ville libre par la Prusse à l'été 1866. Ce n'est que dans les années 1877-1879 que le projet vit finalement le jour, après qu'une association de bourgeois visant à soutenir sa création avait été fondée. Dans sa première conception, le musée se rapportait presque exclusivement à l'époque précédant l'annexion de 1866 : il était un « lieu de mémoire » romantique de la ville d'Empire et de la ville libre, avec, au centre, une mise en scène esthétique de l'arsenal militaire de celle$\mathrm{ci}^{3}$.

3 Avec le départ de la génération des fondateurs, en 1907, commença une nouvelle phase dans l'histoire du musée : la scientifisation du travail du musée et de son personnel alla de pair avec un positionnement dans un conflit qui divisa la ville en deux camps pendant de nombreuses décennies. À partir de 1900, la vieille ville de Francfort devint en effet l'objet des urbanistes. Dans les années 1920, cela aboutit à une véritable lutte de camps. D’un côté, on trouvait les "modernistes ", représentés par l'oberbürgermeister en fonction, Ludwig Landmann, et son urbaniste Ernst May, qui prônaient, depuis 1924, la démolition généralisée et la reconstruction moderne du quartier de la vieille ville, comptant plus de deux mille bâtiments médiévaux et baroques. De l'autre côté, se forma un mouvement de résistance, à l'initiative de bourgeois plus traditionalistes, réunis autour de "l'association des amis de la vieille ville ", fondée en 1922. Ce mouvement voyait en cette dernière un véritable monument et encouragea ainsi sa "guérison »" Dans ce conflit, le musée se situa du côté de la tradition et investit, jusqu'à la fin des années 1930, six bâtiments dans le centre historique. À l'époque nazie, encore, la vieille ville resta le point de référence majeur du travail du musée. Même si le régime nazi le renomma en 1934 «Musée d'histoire de la ville ", il ne parvint pas à imposer, avant la fermeture du musée en 1941 du fait de la guerre, une nouvelle conception du musée comme « musée de la patrie $»^{5}$.

4 Après la Seconde Guerre mondiale, l'institution, de nouveau appelée "musée historique ", se transforma sous son premier directeur Gerhard Bott (1955-1960) en un musée d'art et d'arts décoratifs de la région. L'histoire et le patrimoine régional souffraient alors du poids de la politique du musée menée par le national-socialisme. À ceci s'ajouta le déplacement du musée sur son emplacement actuel, au niveau du Saalhof, à l'extrémité sud de la colline du Römer. Dès 1937, les collections archéologiques avaient été mises à part et formaient le "musée d'histoire régionale préhistorique et antique » (aujourd'hui musée archéologique de Francfort). La construction annoncée pour 
l'ouverture de la Saalhof en 1955 fut certes planifiée au début des années 1960, mais ne fut cependant jamais réalisée. C'est seulement dans les années 1970 qu'un nouvel élan fut donné au projet, grâce à une importante aide financière de la ville. La construction en béton, caractéristique, assez brutale dans sa forme, signalait, dès l'extérieur, que le musée ne voulait pas être un " temple des muses », comme, par exemple, le bâtiment construit en 1877 pour l'institut d'histoire de l'art du Städel. Il voulait au contraire être un « lieu de savoir ", comme une école ou une bibliothèque municipale. L'organisation du musée, qui en résulta, présentait l'histoire allemande dans la perspective de Francfort et, pour la première fois, la commentait et l'expliquait dans son ensemble. Un système d'information, adapté aux espaces d'expositions modernes, avec des pictogrammes, conçu par la grande école de design d'Ulm (Herbert W. Kapitzki) formait l'arrière-plan de toutes les expositions ( documentation historique »). Une lumière au néon, volontairement dénuée d'«aura", créait une atmosphère sobre et neutre. La science historique « critique » des années 1970 influa sur le choix des thèmes, comme sur les informations proposées pour la première fois de façon détaillée. Le musée se définissait comme un " musée pour la société démocratique » et réagissait à " l'état d'urgence de la formation ", tant évoqué à la fin des années 1960. La proximité des concepteurs du musée avec les mouvements sociaux de l'époque, en particulier les mouvements étudiants et féministes, constitua un élément important de cette nouvelle orientation du musée, tant sur le plan social que sur celui du programme ${ }^{6}$.

Dès lors, le musée constitua autant un objet d'attention qu'un objet de controverses dans toute l'Europe, que ce soit pour la nouvelle exigence promue en terme de formation, que pour le positionnement du musée, tout à la fois héritier des Lumières et de la pensée critique. Avec la création de la rive aux musées, en 1977, et sa transformation après 1980, la situation du bâtiment sur le Römerberg changea. La ville ne construisit pas seulement de nouveaux bâtiments pour d'anciens musées (par exemple le nouveau bâtiment du Musée des arts décoratifs, de Richard Meier, ouvert en 1985), mais il fonda également de nombreux nouveaux musées, comme le Musée allemand du film, le Musée allemand d'architecture (créés tous deux en 1984), le Musée juif (1988), agrandi, en 1992, avec le musée de la Judengasse. Le Musée de la poste (1958) devient le Musée de la communication (1990). Toutes ces nouvelles créations furent faites, plus ou moins totalement, à partir des collections du Musée historique de Francfort. Ces musées prirent dorénavant pour thème des aspects importants de l'histoire de la ville de Francfort. Avec la rive aux musées, le Musée historique ne fut plus un «musée universel » pour la culture de la ville, comme cela était prévu au moment de sa fondation, mais devint un musée spécialisé dans l'histoire locale.

6 Cependant, même cette « niche » ne resta pas son terrain exclusif, dans la mesure où les archives de la ville furent renommées "Institut pour l'histoire de la ville», et proposèrent des expositions et des collections très complètes. L'exigeante architecture des nouveaux bâtiments sur la rive aux musées, ainsi que l'évolution de la muséographie, à partir de la fin des années 1970, finirent de mettre le musée historique à la marge.

7 Depuis le début du $\mathrm{XXI}^{\mathrm{e}}$ siècle, le musée historique est de nouveau reconstruit et réaménagé. La nécessité de procéder à des travaux d'assainissement dans l'ancien bâtiment et dans l'édifice en béton a ouvert la voie, à partir de 2005, à deux vastes projets de travaux qui, pour la première fois depuis les grandes transformations de 1972, offraient la possibilité de rénover et de repenser le musée. De 2008 à 2012, le cabinet d'architecture Diezinger (Eichstätt) travailla sur l'ensemble historique du Saalhof. Il 
transforma avec beaucoup de soin cette construction à caractère historique, l'une des plus anciennes de la ville, en un «musée-site » dédié à l'histoire de la ville du XII ${ }^{\mathrm{e}}$ au XX siècle ${ }^{7}$. Lors du concours international lancé pour la reconstruction du musée en 2007-2008, c'est le projet de Lederer Ragnarsdòttir Oei (LRO Architekten, Stuttgart) qui fut choisi. Après une phase de planification de plusieurs années, il est à présent réalisé, depuis 2013, et sera achevé en $2016^{8}$. Depuis 2005, ce projet, dans sa globalité, est lié à l'intérêt renouvelé pour la vieille ville de Francfort - qu'il contribua en même temps à nourrir -, tant de la part du public que des politiques de la ville. La rénovation de l'édifice a suscité, comme déjà dans les années 1920, puis dans les années 1950, un grand débat public sur l'aménagement du coeur historique de la ville. En même temps, il s'agissait, et il s'agit toujours, d'un conflit autour des identités de Francfort: quelles traditions marquent la ville? En quel sens est-elle moderne? Qu'est-ce qui est authentique et véritablement important pour la conscience de la ville elle-même, et qu'est ce qui relève simplement d'une mise en scène, pour le marketing urbain, le tourisme et les sociétés de défense des traditions ?

Dans ce conflit, le musée ne prend plus partie, comme auparavant dans les années 1920, ou dans les années 1950, mais cherche au contraire à devenir un musée pour toute la ville, un musée de la ville. Le retour aux « origines » des années 1860 y aide beaucoup : l'objectif est en effet de faire un musée universel pour la ville du XXI ${ }^{\mathrm{e}}$ siècle, un musée qui reflète la ville dans ses perspectives à la fois historiques, actuelles et futures, et invite au débat. Le musée comme «miroir», comme espace de réflexion de la ville, est devenu l'idée directrice.

9 La nouvelle conception du musée part aussi de l'analyse de la situation actuelle. Nous avons en effet constaté qu'aujourd'hui une part importante des habitants de Francfort était marquée par la diversité culturelle, et ceci bien plus qu'il y a 40 ans. Seule une minorité est ainsi née à Francfort, tandis qu'environ $43 \%$ des habitants possèdent « un passé de migration », et $25 \%$ n'ont pas la nationalité allemande. Ces indicateurs révélant une "superdiversité » constituent, du point de vue du musée, un argument fort pour justifier la nécessité d'établir un lieu culturel où la population urbaine peut s'entendre autour d'un thème unique et commun : leur ville ${ }^{10}$.

10 Dans le même temps, le tourisme global et national a fortement augmenté à Francfort. En 2005, environ $25 \%$ des visiteurs du musée venaient de l'étranger. Parmi eux, le nombre de visiteurs venant d'Asie et d'Amérique s'est particulièrement accru. Le rôle, en arrièreplan, de la plate-forme aérienne de Francfort-sur-le-Main est ici important. Par ailleurs, en $2005,20 \%$ des visiteurs allemands venaient de l'extérieur de la région. Et cette proportion est amenée à augmenter plus qu'à décliner, dans la mesure où Francfort est devenue une destination pour le tourisme urbain à l'intérieur de l'Allemagne, dépassant notamment Cologne. La majeure partie de nos visiteurs, environ $55 \%$, venaient enfin, en 2005, de Francfort ou de sa région ${ }^{11}$. La relation entre les visiteurs extérieurs et les " experts de la ville», c'est-à-dire les habitants de la région, est ainsi globalement équilibrée. C'est à ces deux groupes de visiteurs que le nouveau Musée historique de Francfort veut s'adresser. La conséquence en est que le musée se doit de proposer une offre diverse qui s'adresse autant aux personnes qui visitent Francfort pour la première fois - quand ils ne découvrent pas l'Europe - qu'aux experts de leur ville.

11 Plus profondément encore que les mouvements de migration et les dynamiques du tourisme, c'est aussi le processus d'urbanisation globale qui a changé la situation des « anciennes » villes européennes et celle de leur musées. Francfort est cependant la seule 
ville d'Allemagne qui est concernée par ce phénomène depuis déjà plusieurs décennies. Avec ses bourses et ses banques, sa foire et son aéroport, la ville est en effet marquée, bien plus que les autres villes allemandes, par son importante inscription dans les réseaux globaux depuis la fin de la seconde guerre mondiale. Avec le changement de siècle, le rôle de la ville, en tant que pôle majeur dans le réseau global, s'est encore accru. C'est pour cela que la sociologue américaine, spécialiste de la ville, Saskia Sassen, a caractérisé Francfort comme la seule " global city » d'Allemagne ${ }^{12}$. Dans ces métropoles en réseaux, les surfaces urbaines perdent très vite de leur caractère: l'architecture, les moyens de transports, les objets de consommation, les moyens de communication tendent à se ressembler toujours davantage les uns les autres. Le personnel des secteurs de service, très inscrits dans ces réseaux, passe de ville en ville, contribuant à faire s'émousser les spécificités de celles-ci. La construction d'une identité urbaine, fondée sur des récits partagés et sur des espaces communs, est rendue plus difficile dans ce contexte : sa force de lien diminue. En transformant quelque peu le sens d'un concept courant de la recherche sociologique récente, on pourrait ainsi parler d'un déclin de la «logique interne » de nos villes ${ }^{13}$.

12 La puissance de conviction et la force des biens culturels transmis, associés aux récits ou aux termes canoniques qui leur sont liés, tels que "patrimoine", "héritage " ou "traditions", dépendent toujours en grande partie du niveau de formation et des origines sociales. Aujourd'hui, dans la mesure où nous vivons à une époque de grande diversité culturelle, il serait fatal que les musées transmettent simplement et sans recul ces éléments du patrimoine culturel, hérités de génération en génération. Les musées courraient alors le danger d'être des «niches » d'une élite lettrée locale, et de perdre ainsi toute pertinence. En tout cas, ils ne seraient plus en situation de toucher les sociétés urbaines du XXI e siècle, caractérisées par leur hétérogénéité culturelle.

De façon paradoxale, le développement de la diversité et de l'hybridité culturelles dans le cadre de l'urbanisation globale peut aussi être caractérisé comme une "glocalisation ", au sens où l'émergence localisée de nouveaux « mélanges ", faits de diversités et d'hybridité, contribuent en retour à la spécificité locale ainsi qu'à la création de nouvelles formes de traditions ${ }^{14}$. D'un autre côté, la glocalisation a eu comme conséquence la diminution des relations au-delà des villes, en particulier au niveau national, au profit de la communauté du lieu même. La vie de la communauté du lieu et la spécificité locale, avec ses particularités culturelles et historiques, deviennent ainsi plus importantes que le fait de s'entendre sur des valeurs ou des histoires nationales. Et cette spécificité est d'autant plus exprimée, et devient d'autant plus l'objet de discussions et de "négociations », qu'un nombre important de gens n'est pas né dans leur lieu de résidence. Il s'agit là d'une grande chance pour les musées de ville. Car ils peuvent devenir des lieux importants dans les débats autour de la vie en commun, des images de la ville, et naturellement autour des représentations communes des notions de "tradition ", d' " héritage », et d' " histoire ", comme du même et de l'autre.

La façon dont le musée de la ville du XXI ${ }^{\mathrm{e}}$ siècle s'adresse à ses propres habitants devrait rendre possible cette conciliation. Dans la société du savoir du XXIe siècle, la connaissance experte des utilisateurs ( users ») joue un rôle totalement nouveau, rendu possible par les structures participatives et collaboratives du web 2.0. Wikipedia, les contenus générés par les utilisateurs (" user generated content»), la collaboration ouverte («crowd sourcing ») et d'autres plates-formes ou techniques de mise en réseau, ont aussi des effets sur les institutions du savoir, comme les musées. Davantage de visiteurs veulent devenir des 
«usagers » et prendre une part active au travail du musée, commenter le travail du musée ou même y contribuer activement ${ }^{15}$.

Pour la nouvelle conception du Musée historique de Francfort, il s'ensuit que celui-ci cherchera à l'avenir à toujours plus s'associer aux initiatives de la population de la ville ou de la région. Davantage d' " interfaces » avec le public urbain ou régional doivent être créées en ce sens, afin de rendre cela possible et durable. Par cette ouverture, le musée doit devenir un lieu de présentation et de discussion de perspectives différentes, qu'elles soient scientifique, culturelle, artistique, individuelle ou engagée. Mais ce sont aussi des thèmes controversés de la société urbaine qui doivent pouvoir être débattus et présentés ici.

En résumé, les objectifs du nouveau Musée historique de Francfort peuvent être formulés ainsi :

1. Le musée ne raconte pas seulement l'histoire de la ville, il doit discuter aussi son présent et son avenir.

2. Il aide les visiteurs du monde entier à comprendre Francfort.

3. Pour les habitants de Francfort et de la région, le musée est un forum intéressant pour tous les thèmes urbains. Il les aide à mieux re-« lire » leur ville.

4. Le musée implique activement ses visiteurs : les visiteurs peuvent devenir des « usages » (en anglais « users ») du musée.

5. Le musée offre une diversité de regards sur Francfort : sous différentes formes, il se tourne vers les différents besoins des visiteurs, avec des offres particulières pour les jeunes visiteurs dans toutes les expositions (« Musées pour les familles »).

De ce fait, au lieu d'un grand "récit », on doit trouver dans le nouveau Musée historique de Francfort une multitude d'expositions ou d'offres qui se croisent dans les thèmes de la ville. Le musée se présente dorénavant en "quartier-musée », comme un miroir de la ville sous toutes ses facettes. Déjà la grande différence entre, d'un côté, les touristes et les visiteurs de Francfort, comptant un nombre important de voyageurs originaires d'Asie ou d'Amérique, et d'autre part, les experts de la ville, habitants de la ville et de la région, oblige à une diversification de l'offre. Les connaissances préalables des visiteurs comme leurs besoins (rapport budget-temps, informations, terrain) divergent ici à l'extrême ; aussi n'est-il plus possible d'y répondre dans le cadre d'une grande et unique exposition. La part croissante de touristes étrangers parmi les visiteurs du musée offre aussi la chance de développer les offres explicatives qui, dans le même temps, peuvent aussi correspondre aux besoins de la population de la ville, toujours plus diverse.

La référence délibérée aux thèmes du temps présent dans les nouvelles expositions durables, en premier lieu «Frankfurt JETZT! » («Francfort maintenant!») ou dans les modules de « Frankfurt EINST ?» (« Francfort jadis?»), doit aussi contribuer à diminuer les barrières du savoir et les barrières culturelles pour les visiteurs du musée. Une autre stratégie du nouveau Musée historique de Francfort est de concevoir le musée dans son ensemble avec un « jeu de piste » pour les jeunes visiteurs. Par la dimension interactive de ses offres, le musée vise ainsi à s'adresser aux jeunes visiteurs, entre 6 et 13 ans, et à leurs accompagnateurs (parents, grands-parents). Comme l'expérience le prouve, ces offres sont très attractives pour tous les visiteurs, parce qu'elles sont facilement accessibles. Dans cette visée, les "espaces atelier ", qui se trouvent directement dans les expositions, offrent à la fois aux utilisateurs actifs des collections et aux groupes de travail des lieux pour chercher. Ces offres (jeu de piste pour les enfants ou ateliers) ont été conçues par Le musée des enfants de Francfort, qui joue dorénavant un rôle actif dans 
l'agencement d'ensemble du musée. L'expertise de ce service, fondé dès 1972, est, pour la nouvelle conception du musée, de la plus haute importance.

Dans ce texte, j'évoquerai quatre éléments qui thématisent, chacun à leur façon, les différentes identités de Francfort. Avant même d'entrer dans le musée, les visiteurs sont confrontés à une installation inhabituelle. À travers une ouverture, prenant la forme d'une fontaine, située sur le nouvel emplacement du musée, le regard plonge au niveau sous-terrain. Là se trouve une grande "boule à neige", conçue comme symbole du " souvenir ", image la plus stéréotypée de la ville. L'idée de cette installation a été conçue par les scénographes de Kossmann Dejong à Amsterdam. Ici l'idée n'est pas seulement d'offrir un "souvenir » à Francfort, mais huit en même temps. Le message est que « Francfort est plusieurs villes ». Les visiteurs peuvent choisir sur la "boule à neige » un cliché : apparaitt alors, grâce à un robot, un modèle urbain d'une taille de 2,5 mètres sous la coupole de verre, tandis que commence un film Multivision de deux minutes projeté sur les murs. Les modèles, conçus par des artistes, montrent sur chacun des clichés une sélection de bâtiments et d'événements de l'histoire de Francfort ou du temps présent. Les clichés ou les images identitaires prévus pour le moment sont: "Bankfurt » (ou «Mainhattan », la ville de l'argent, la place financière), «Krankfurt » (la capitale de la criminalité), le carrefour (les noeuds de circulation), «Big Eppel » (la ville où il fait bon vivre, la ville des mécènes), la ville critique (la métropole des contestations), la ville du livre (ville de la foire du livre, des éditions, des journaux, des écrivains), «DC » (la ville secrète), la ville de l'industrie (« capitale du travail »).

Cette évocation des multiples identités de la ville touche le visiteur, qu'il vienne de l'étranger ou qu'il soit de la région. Elle s'appuie en effet sur les clichés qu'il a déjà en tête, ou qui sont diffusés dans les guides de voyage ou les autres médias. Ils peuvent aussi évoquer quelque chose d'oublié ou de refoulé. Ici se cachent des éléments identitaires, qui souvent remontent loin dans l'histoire de la ville. Le déploiement de cette interprétation artistique et l'utilisation du monde visuel de la multivision donnent ainsi à voir en un bref laps de temps un premier panorama de la ville. Par là même, les clichés se tempèrent et s'éclairent mutuellement. En vingt minutes environ, les visiteurs peuvent avoir un aperçu inhabituel de l'histoire de Francfort et de son présent, et ainsi saisir que les forts contrastes et les contradictions de cette ville, aussi fascinantes qu'irritantes, sont justement la clé pour comprendre ce qui fait sa spécificité.

Dans les grandes unités d'exposition du nouveau musée, « Francfort jadis ? 800 d'histoire de la ville », l'histoire de Francfort est présentée sur 2000 mètres carrés et en quatre galeries thématiques. Trois d'entre elles portent plus particulièrement sur les traits spécifiques de la ville, qui la caractérisent depuis le XII ${ }^{e}$ siècle. Après l'introduction dans la galerie « Images de la ville », le visiteur atteint la galerie "ville citoyenne ». Là, il est question de la ville comme république citoyenne. Un grand mur donne à voir près de 200 portraits, ouvrant sur différentes biographies parmi lesquels les visiteurs sont invités à opérer un choix : les biographies choisies doivent ensuite être présentées sous la forme de petite exposition temporaire, dans l'un des huit cabinets biographiques de la galerie thématique. De cette façon, de nouveaux contenus apparaitront toujours dans le cadre de l'exposition permanente. La troisième partie de la section « ville citoyenne » concerne les groupes de la société urbaine : les confréries, les métiers, les corporations, les loges, les sociétés de lecture, les associations, les sociétés d'utilité publique, jusqu'aux initiatives citoyennes d'aujourd'hui. 
21 Dans la galerie thématique qui suit, « la ville de l'argent », il est question de Francfort comme place financière et comme ville du commerce, depuis le Moyen Âge. Le visiteur est d'abord confronté à une grande « image de monnaie ». Depuis le denier de Charlemagne, qui a été introduit à Francfort en 794, jusqu'à l'euro en 2000, ce sont en effet près de 4000 pièces de monnaie qui ont été diffusées. Tout ceci forme un panorama de l'empreinte de la monnaie en Allemagne, sur 1200 ans d'histoire. Les visiteurs peuvent trouver les monnaies de leur lieu d'origine, «naviguer » dans le monde des images et des signes de la monnaie, et apprendre à comprendre la fonction d'une bourse au XVI ${ }^{\mathrm{e}}$ et au XVII ${ }^{\mathrm{e}}$ siècle, à l'époque où celle de Francfort fut fondée. Une autre installation se concentre sur les significations de l'argent à travers huit installations : des concepts comme "crédit » ou " confiance ", " compte ", « intérêt ", « économie ", "spéculation », mais aussi " salut de l'âme » ou "réparation », sont illustrés par d'impressionnants ensembles d'objets liés à l'histoire de Francfort. À la fin de la galerie, se trouve une installation interactive : sur une forme de «bascule ", les visiteurs peuvent, par un mouvement de balancier, révéler les périodes de crise et d'expansion de la place financière de Francfort, et les approfondir par les pièces d'exposition.

La dernière galerie thématique est consacrée à la "ville monde ». Le visiteur est alors comme emporté par différents moyens de transport de la ville. Au centre, se trouvent deux « carrousels » : la ville de l'élection du roi se situe à côté de la ville de la première assemblée nationale; la cathédrale impériale jouxte l'église Saint-Paul. Le visiteur peut approfondir l'histoire de l'élection et du couronnement de l'empereur, ses effets sur l'histoire de la ville, et ainsi apprendre à mieux connaître la tradition monarchique et démocratique de la ville. Par là-même, Francfort a été longtemps une plaque tournante politique ou une « capitale invisible » de l'Allemagne. La fin porte sur le rôle central que Francfort joue, jusqu'à nos jours encore, comme ville de l'édition et du commerce du livre, et comme point nodal dans le domaine de la communication. L'exposition «Francfort, jadis?» a été développée en étroite collaboration avec l'équipe de scénographes de Gillmann et Schnegg de Bâle.

23 À l'étage supérieur du grand espace d'exposition, au dessus de «Frankfurt, Einst?» («Francfort, jadis ? ) se trouve le « laboratoire » de la ville, « Frankfurt jetzt! Die Stadt von heute und morgen» ("Francfort maintenant! La ville d'aujourd'hui et de demain»), entouré par plus de 80 fenêtres sur la cité. Dans cette grande exposition d'environ mille mètres carrés, il est aussi question des identités de Francfort. En ouverture, on trouve un grand modèle de la ville d'aujourd'hui, d'environ 70 mètres carrés, où les visiteurs peuvent diffuser leurs propres images, films ou textes. Au préalable, les visiteurs du musée sont invités à marquer les lieux importants de la ville de leur point de vue. Le modèle fonctionne comme un élément d'exposition interactif, qui rend possibles des découvertes étonnantes, aussi bien par un mode d'approche " analogique » que par des moyens de connaissance digitaux. À l'autre bout de l'espace (à l'est), se trouve la "bibliothèque des anciens ", forme $d^{\prime}$ ' archive ouverte " rassemblant des contributions et des collections d'objets de Francfortois et Francfortoises sur leur ville. Là, Francfort est envisagée à travers différentes perspectives individuelles. Pour le moment, il y a 70 auteur-e-s, à l'avenir il y en aura plus de 150. Au milieu de l'espace, ont lieu, deux à trois fois par an, des expositions temporaires. Ici le "laboratoire de la ville " réalise constamment de nouveaux projets d'exposition, en étroite coopération avec les initiatives de la population Depuis 2011, ceci a déjà eu lieu, en différents endroits de la ville, sous la forme du «laboratoire hors les murs» (2011 «Ostanfang/Ostende», 2012, " Mein 
Stadionbad», 2013, "G-Town Ginnheim »). Sous cette forme innovative, il s'agit de développer et de concevoir des expositions fondées sur la participation des profanes ou des « expert-e-s de la ville », et de pouvoir ainsi rassembler des expériences ${ }^{16}$. Toutes les expositions seront rassemblés dans la section "archive des projets", et rendues disponibles aux visiteurs sous la forme de documentations. Ainsi se déploiera un panorama de la ville, du point de vue de différents groupes et à partir de différents thèmes. Partant du présent de la ville, les regards pourront se tourner vers l'histoire et vers l'avenir.

Les autres offres permanentes du musée tournent également autour des spécificités de la ville: «Les collectionneurs et mécènes de Francfort » raconte l'histoire de la ville à travers les fondations financées par des collectionneurs privés et par des habitants de la $v$ ille ${ }^{17}$. L'exposition « Le temps des Staufen » située dans une zone archéologique située au niveau des soubassements, mène les spectateurs aux XII ${ }^{\mathrm{e}}-\mathrm{XIII}{ }^{\mathrm{e}}$ siècles, période essentielle dans l'histoire de la ville. Le «panorama du Main» dans la Rententurm montre l'importance de l'ancien port sur le Main et de la porte la plus importante de la ville, le Fahrtor. En 2015/16, le « musée des enfants » retournera dans le musée rénové sur le site du Römer, et marquera le souci de proposer des offres aux jeunes visiteurs et aux familles dans l'ensemble du musée.

\section{NOTES}

1. Voir Mandy Nauwelarts (dir.), The Future of the Past : reflections on history, urbanity and Museum, Antwerpen 2000 ; Renée Kistemaker (dir.), City museums as centres of civic dialogue?, Amsterdam : Renée Kistemaker (Amsterdam Historical Museum) 2006 ; Claudia Gemmeke, Franziska Nentwig (dir.), Die Stadt und ihr Gedächtnis. Zur Zukunft der Stadtmuseen, Bielefeld : transcript, 2011.

2. Voir Hans-Ulrich Thamer, «Vom Heimatmuseum zur Geschichtsschau. Museen und Landesaustellungen als Orte der Erinnerung und Identitätsstiftung », Westfälische Forschungen, $\mathrm{n}^{\circ}$ 46, 1996, p. 429-448; Jan Gerchow, «Museen », in : Michael Maurer (dir.), Aufriß der Historischen Wissenschaften, Bd. 6: Institutionen, Stuttgart: Reclam, 2002, p. 316-400; id., "Stadt- und regionalhistorische Museen ", in: Bernhard Graf, Volker Rodekamp (dir.), Museen zwischen Qualität und Relevanz. Denkschrift zur Lage der Museen, Berlin : G-+-H-Verl. (Berliner Schriftenreihe zur Museumsforschung, 30), 2012, p. 341-348.

3. Voir Jürgen Steen, « Das Historische Museum Frankfurt am Main - Plan, Gründung und die ersten fünfundzwanzig Jahre», in: Trophäe oder Leichenstein. Kulturgeschichtliche Aspekte des Geschichtsbewußtseins in Frankfurt im 19. Jahrhundert, Frankfurt am Main : Histor. Museum (Kleine Schriften des Historischen Museums, 12), 1978, p. 23-48.

4. Voir Das Frankfurter Altstadtmodell der Brüder Treuner, hrsg. von Jan Gerchow und Petra Spona (Kunststücke des historischen museums frankfurt, Bd. 1), Frankfurt, 2011.

5. Voir Almut Junker, « Universale Pläne und ihr Scheitern - das Historische Museum Frankfurt 1870-1920 ", in : Die Zukunft beginnt in der Vergangenheit. Museumsgeschichte und Geschichtsmuseum, Frankfurt am Main : Amt für Wiss. u. Kunst (Schriften des Historischen Museums Frankfurt am Main 16), 1982, p. 236-259. Jürgen Steen, «Zwischen bürgerlicher Tradition und 
weltanschaulicher Integration. Die Geschichte des Historischen Museums Frankfurt am Main im 3. Reich ", in : op. cit., p. 274-308.

6. Voir Detlef Hoffmann, Almut Junker, Peter Schirmbeck (dir.), Geschichte als öffentliches Ärgernis, oder: Ein Museum für die demokratische Gesellschaft, Fernwald, Wissmar: Anabas-Verlag Kämpf, 1974. La publication de Manfred Kittel, Marsch durch die Institutionen? Politik und Kultur in Frankfurt am Main nach 1968, München: Oldenbourg (Quellen und Darstellungen zur Zeitgeschichte, hrsg. vom Institut für Zeitgeschichte 86), 2011, p.101 et p.125, ne poursuit malheureusement que la critique conservatrice du musée développée dans les années 1970. Il n'évoque pas la portée muséologique de la conception de l'institution, ni les discussions de l'époque.

7. Voir les articles de Norbert Diezinger et de Jan Gerchow, in: Die Renovierung des Frankfurter Saalhofs, hrsg. vom historischen museum frankfurt (CURA 2012), Frankfurt am Main 2012 ; Enrico Santifaller, Historisches Museum Frankfurt (Baukulturführer 67), Amberg : Büro Wilhelm, 2012.

8. Voir Arno Lederer, Jórunn Ragnarsdóttir, Marc Oei, «Die Neubauten für das historische museum frankfurt», in: CURA 2011, hrsg. von Jan Gerchow, historisches museum frankfurt, Frankfurt am Main, 2011, p. 12-21.

9. Voir Wolfgang P. Cilleßen, Jan Gerchow, Die Baudenkmäler des historischen museums frankfurt, in : CURA 2010, hrsg. von Jan Gerchow, historisches museum frankfurt, Frankfurt am Main, 2010, p. 4-27.

10. En ce qui concerne les chiffres, voir le rapport Vielfalt bewegt Frankfurt. Integrations- und Diversitätskonzept für Stadt, Politik und Verwaltung: Grundsätze, Ziele, Handlungsfelder, Magistrat der Stadt Frankfurt am Main 2010 -

http://www.frankfurt.de/sixcms/media.php/738/

Integrationskonzept_Magistrat_06_10.1557580.pdf ; en ce qui concerne les conséquences pour la conception du musée voir Jan Gerchow, «Stadtmuseen im Zeichen der Globalisierung. Positionen für die Neukonzeption des historischen museums frankfurt ", Museumskunde, 77-2, 2012, p. 55-59.

11. Voir «Besucherströme an ausgewählten Kultureinrichtungen im Planungsverband Ballungsraum Frankfurt/Rhein-Main, Ergebnisse der Sondererhebung im Januar/Februar 2005 ", Statistik Hessen, 04/2005, p. 54 et suiv.

12. Voir Saskia Sassen, Metropolen des Weltmarktes. Die neue Rolle der Global Cities, Frankfurt am Main/New York : Campus, 1996.

13. Le concept de « logique interne des villes » a été forgé par Helmuth Berking et Martina Löw, afin d'interroger la ville - généralement envisagée en sociologie comme un phénomène social total, à valeur universelle - d'une façon plus individualisée et différenciée. Voir Helmuth Berking, Martina Löw (dir.), Die Eigenlogik der Städte. Neue Wege für die Stadtforschung, Frankfurt am Main/New York : Campus (Interdisziplinäre Stadtforschung, 1), 2008.

14. Voir Roland Robertson, "Glokalisierung. Homogenität und Heterogenität in Raum und Zeit ", in: Ulrich Beck (dir.), Perspektiven der Weltgesellschaft, Frankfurt am Main: Suhrkamp, 1998, p. $192-220$.

15. Voir le texte très discuté de James Surowieck, The wisdom of crowds. Why the many are smarter than the few and how collective wisdom shapes business, economies, societies and nations, New York 2004. En relation avec les musées, voir : Susanne Gesser, Angela Jannelli, Sibylle Lichtensteiger, Martin Handschin (dir.), Das partizipative Museum. Zwischen Teilhabe und User Generated Content. Neue Anforderungen an kulturhistorische Ausstellungen, Bielefeld : transcript, 2012.

16. Voir Ostend // Ostanfang, Dokumentation der Ausstellung des „stadtlabor unterwegs“, historisches museum frankfurt, Frankfurt am Main 2011; Mein Stadionbad - eine Ausstellung mit Schwimmbad, historisches museum frankfurt, Frankfurt am Main 2012.

17. Voir Anja Johann (dir.), Frankfurter Sammler und Stifter, Frankfurt am Main: Henrich Ed. (Schriften des historischen museums frankfurt, 32), 2012. 


\section{AUTEURS}

\section{JAN GERCHOW}

Musée historique de Francfort 